ARTICLE

\title{
Systematic comparison of sea urchin and sea star developmental gene regulatory networks explains how novelty is incorporated in early development
}

\author{
Gregory A. Cary (10) 1,3,5, Brenna S. McCauley 1,4,5, Olga Zueva', Joseph Pattinato', William Longabaugh ${ }^{2}$ \& \\ Veronica F. Hinman (D) ${ }^{1 凶}$
}

The extensive array of morphological diversity among animal taxa represents the product of millions of years of evolution. Morphology is the output of development, therefore phenotypic evolution arises from changes to the topology of the gene regulatory networks (GRNs) that control the highly coordinated process of embryogenesis. A particular challenge in understanding the origins of animal diversity lies in determining how GRNs incorporate novelty while preserving the overall stability of the network, and hence, embryonic viability. Here we assemble a comprehensive GRN for endomesoderm specification in the sea star from zygote through gastrulation that corresponds to the GRN for sea urchin development of equivalent territories and stages. Comparison of the GRNs identifies how novelty is incorporated in early development. We show how the GRN is resilient to the introduction of a transcription factor, pmar1, the inclusion of which leads to a switch between two stable modes of Delta-Notch signaling. Signaling pathways can function in multiple modes and we propose that GRN changes that lead to switches between modes may be a common evolutionary mechanism for changes in embryogenesis. Our data additionally proposes a model in which evolutionarily conserved network motifs, or kernels, may function throughout development to stabilize these signaling transitions.

\footnotetext{
${ }^{1}$ Department of Biological Sciences, Carnegie Mellon University, Pittsburgh, PA 15213, USA. ${ }^{2}$ Institute for Systems Biology, Seattle, WA, USA. ${ }^{3}$ Present address: The Jackson Laboratory, Bar Harbor, ME, USA. ${ }^{4}$ Present address: Huffington Center on Aging, Baylor College of Medicine, 1 Baylor Plaza, Houston, TX 77030, USA. ${ }^{5}$ These authors contributed equally: Gregory A. Cary, Brenna S. McCauley. ${ }^{凶}$ email: vhinman@andrew.cmu.edu
} 
T he regulatory program that controls development is unidirectional and hierarchical. It initiates with early asymmetries that activate highly coordinated cascades of gene regulatory interactions known as a gene regulatory network (GRN). GRNs function to orchestrate the intricate cellular and morphogenic events that comprise embryogenesis ${ }^{1,2}$, and their topologies must be structured in ways that permit the robust development needed to reliably produce viable embryos. While genetic variation can arise anywhere in the genome and affect any part of an individual GRN, the need to form a viable embryo provides a constraint on the types of variation that pass the filter of selection. The timing and mechanisms of potential developmental constraint persist as topics of intense debate ${ }^{3-7}$, which can only be resolved by systems-level comparisons of experimentally established GRNs. The evolution of transcription factors (TFs) used in early development presents an especially intriguing problem in the context of maintaining developmental stability ${ }^{8}$.

The GRN for the specification of sea urchin endomesoderm is the most comprehensive, experimentally derived GRN known to date $^{9-11}$. It explains how vegetal-most micromeres express signaling molecules, including Delta, needed to specify the adjacent macromere cells to endomesoderm, how micromeres ingress as mesenchyme, and are finally specified to form a biomineralized skeleton. This GRN initiates with the maternally directed nuclearization of $\beta$-catenin, which activates the paired homeodomain TF pmar ${ }^{12}$. Pmarl represses the expression of hesC. The HesC $\mathrm{TF}$ is a repressor of genes encoding many of the TFs needed to specify micromere fate (i.e., $a l x 1$, ets $1, t b r$, and $t e l$ ) including the delta gene. The activation of Pmar1, therefore, indirectly leads to the expression of many of the regulatory genes within the vegetal pole, micromere territory in what has been termed the doublenegative gate ${ }^{13}$. The Pmar1 TF appears to be a novel duplication of the phb gene, and is found only in sea urchins ${ }^{14}$. No clear ortholog of pmar1 exists in available genomes or transcriptomes from sea stars (Patiria miniata and Acanthaster planci), brittle stars (Amphiura filiformis), or hemichordates (Saccoglossus kowalevskii $^{15-19}$ and thus the Pmar1 repression of hesc, i.e., the double-negative gate ${ }^{13}$, functions only in modern sea urchins.

In this work, we assemble a detailed GRN for sea star endomesoderm specification through gastrulation (Supplementary Fig. 1). Understanding the impact of integrating novelty into early development demands such a systems-level approach: not one limited to local properties around the new circuit, but an understanding of how the network as a whole responds to the change. An interactive, temporal model, including primary and published data, is hosted on a web server (grns.biotapestry.org/ PmEndomes), which allows for further and more fine-grained exploration (Supplementary Fig. 2). This GRN was produced using the same experimental approaches as those used to generate the sea urchin network ${ }^{20}$ to allow for a meaningful comparison. This sea star GRN, therefore, presents an unprecedented opportunity to compare these networks to understand how they have evolved. Using this network comparison, we show how bimodal switches in signaling pathways permit an evolutionary transition in regulatory network topology, and how such a transition is buffered by the presence of conserved regulatory kernels.

\section{Results}

Delta and $\mathrm{Hes} \mathrm{C}$ are engaged in a canonical regulatory motif. In contrast to the sea urchin ${ }^{13}$ (Fig. 1f), sea star blastula stage embryos co-express the delta and hesC transcripts throughout the endomesoderm-fated vegetal pole (Fig. 1a), but their expression is partitioned into adjacent cells by midway through gastrulation (Fig. 1b). Indeed, in all known species of echinoderms that lack an identifiable pmarl gene, hes $C$ remains expressed within the delta ${ }^{+}$ territory of the blastula mesoderm ${ }^{15,17,21}$. The related Phb genes have recently been shown to act as positive inputs into endomesodermally expressed hesc in sea star and cidaroid sea urchin ${ }^{14}$. We show that Tgif also positively regulates hes $C$ expression specifically in the sea star mesoderm (Fig. 1e). This input is not possible in the sea urchin given that these transcription factors are not co-expressed 22,23 . Not all inputs into hes $C$ are changed, however, as blimp1 (formerly krox), a known repressor of hesC in sea $\operatorname{urchin}^{24}$, also represses sea star hesC (Fig. 1c, d). Thus, the gain of repression by Pmarl and the loss of positive input from Tgif drive the differences in hes $C$ and delta co-expression between sea urchins and sea stars (Fig. 1g). The local impact of integrating pmar1 in the sea urchin network, therefore, is the exclusion of hes $C$ expression from the territory of cells that will express the delta ligand at the vegetal pole. It is via the Delta signal that these cells induce adjacent cells to various endomesodermal fates ${ }^{25-28}$. The early asymmetry in expression of the Delta ligand makes sea urchin micromeres sufficient to induce ectopic endomesoderm when transplanted to the animal pole or to animal blastomeres alone ${ }^{29}$. Thus, Delta induction is critical to specifying mesodermal cell types and is one of the central genes providing the regulatory function of the micromeres in sea urchins.

The co-expression of transcripts encoding Delta, $\mathrm{HesC}$, and the Notch receptor (Supplementary Fig. 3) in the sea star vegetal pole suggests the potential for lateral inhibition regulatory interactions. In many contexts lateral inhibition functions to segregate an equipotential field of cells into distinct cell types ${ }^{30-33}$. Perturbing Delta-Notch signaling and the expression of hes $C$ in sea star blastulae reveals that signaling between adjacent cells through the Notch receptor activates the expression of hes $\mathrm{C}$ (Fig. 2a-c), which in turn represses delta expression (Fig. 2d, e). Thus, we demonstrate that the change in upstream regulation between sea urchin and sea stars that results in co-expression of delta and hesC at blastula stage allows for lateral inhibition (LI) regulatory interactions in sea stars, compared to the inductive mechanism used in sea urchins (Fig. 2h).

Partitioning of mesodermal subtypes involves Delta/Notch lateral inhibition. Given that Delta, Notch, and $\mathrm{Hes} C$ engage in canonical LI, we sought to understand how this process might function in specifying cell types in the sea star mesoderm. The sea star mesoderm originates from the central vegetal pole of the late blastula, a molecularly uniform territory (Fig. 3a, b and Supplementary Fig. 4). During gastrulation this territory sits at the top of the archenteron and segregates into at least two distinct cell types -blastocoelar mesenchyme and coelomic epithelium (Supplementary Fig. 4). These two lineages become molecularly distinct by mid-gastrula stage $(\sim 36 \mathrm{~h})$ and ets 1 expressing mesenchyme cells begin ingressing at $46 \mathrm{~h}$ (Fig. 3c-f). Each ets $1^{+}$cell is generally separated from another $\mathrm{ets}^{+}$cell by two intervening nuclei of $e t s 1^{-}$cells (Fig. $3 \mathrm{~g}$ ) while the intervening cells express six3 (Fig. $3 \mathrm{~h}$ ), a gene that is also initially broadly mesodermal in blastulae, but is later expressed in the coelomic epithelium (Supplementary Fig. 3). ets $1^{+}$cells also express the transcript encoding the Delta ligand (Fig. 3i). From these data we propose a model in which lateral inhibition leads to the restricted expression of ets 1 in the delta ${ }^{+}$cell and six3 in the neighboring cell.

We inhibited Notch signaling to explicitly test the lateral inhibition model. The model predicts that such a treatment would lead to an expansion of the primary cell type (i.e., delta ${ }^{+}$ presumptive mesenchyme) and a concomitant reduction of the secondary cell type (i.e., presumptive coelomic mesoderm). Indeed, inhibition of Notch results in an increase in cells expressing the mesenchyme genes ets 1 and $\operatorname{erg}$ (Fig. 4a-f), and a 

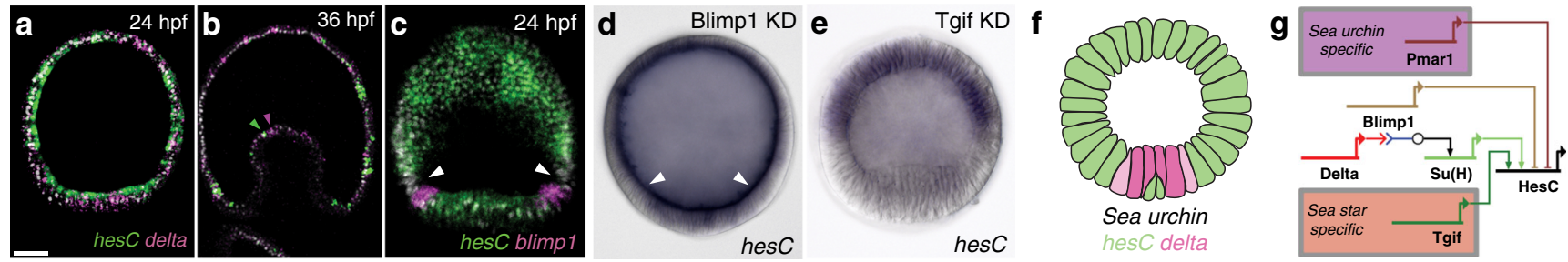

Fig. 1 Sea star hes $C$ is positively regulated downstream of the mesoderm kernel and is co-expressed with delta. Sea star hes $C$ and delta transcripts are co-expressed in the vegetal mesoderm (a) until mid-gastrula stage (b), or 36 hours post fertilization (hpf). Sea star hesC and blimp1 are expressed in partially non-overlapping domains during blastula stage (c) and morpholino knockdown (KD) of blimp1 results in an expansion of the expression domain of hesC (d). Morpholino knockdown of sea star Tgif produces a mesoderm-specific decrease in hesC expression (e). Schematic showing non-overlapping expression domains of sea urchin hes $\mathrm{C}$ and delta transcripts at blastula stage (f). Regulatory inputs to the hes $\mathrm{C}$ gene that are specific to sea urchin embryos, sea star embryos, and those that are common to both (g). Data shown are double fluorescent WMISH showing both hesC expression (green) and either expression of delta or blimp1 (magenta) (a-c) and colorimetric WMISH (d, e). Data are representative of two biologically independent experiments consisting of at least ten embryos each. Scale bar represents $50 \mu \mathrm{m}$; applicable to all images in the panel.

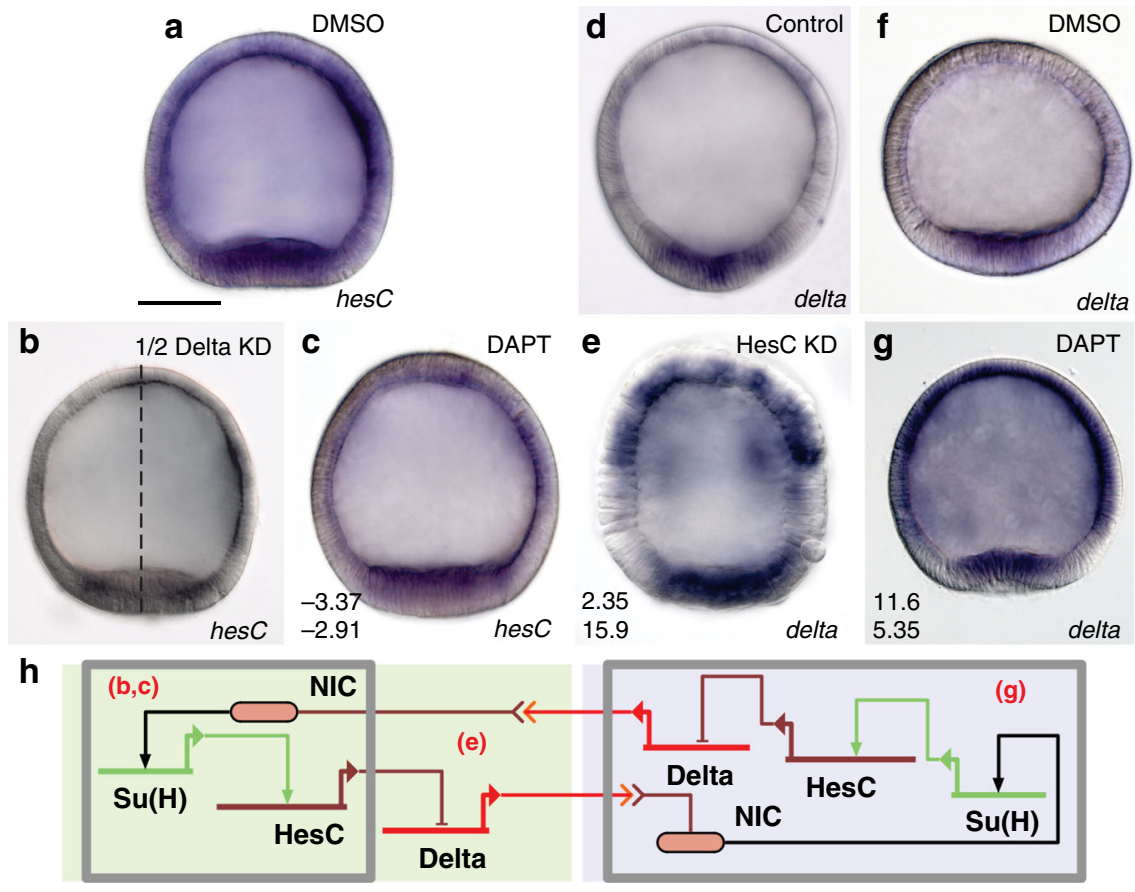

Fig. 2 Testing lateral inhibition of delta and hesC by inhibition of Notch signaling (DAPT) and morpholino knockdown (KD) of HesC. Using DAPT, an inhibitor of the proteolytic gamma-secretase necessary for notch signal transduction, we observe both a down-regulation of hesC (c) and an upregulation of delta transcripts ( $\mathbf{g}$ ). Importantly the down-regulation of hes $C$ is phenocopied by injection of a morpholino targeting the delta transcript into one of the first two blastomeres (b). Knockdown of $\mathrm{Hes} C$ with an antisense morpholino yields an upregulation of both delta (e) and hes $\mathrm{C}$ transcripts. Lateral inhibition network showing relationships tested by previous experiments (h); red letters indicate figure panel above supporting connection. All images are colorimetric WMISH with the probes to the indicated genes. Images are representative of two biologically independent experiments consisting of at least ten embryos each. Scale bar represents $50 \mu \mathrm{m}$; applicable to all images in the panel. Numbers in the lower left corner of (c, e, and $\mathbf{g})$ represent normalized $\log 2$ fold-change values of perturbed expression compared with control (i.e., $\mathbf{a}, \mathbf{d}$, and $\mathbf{f}$ ) as measured by qPCR.

reduction in cells expressing coelomic epithelium genes six3 and pax6 (Fig. 4g-j). Moreover, we also observe a consistent morphological shift with an increase in the number of cells ingressing into the blastocoel from the archenteron and a reduction in the epithelium. These data confirm our hypothesis that the sea star mesoderm partitions through the action of DeltaNotch-mediated lateral inhibition. This also shows a consequence of the changes to the regulation of hes $C$ that allow co-expression with delta-a switch in the mode of Delta-Notch signaling between lateral inhibition when co-expressed and induction when spatially distinct. Asymmetric expression of Delta-Notch regulators typically produces an inductive mode of signaling ${ }^{34}$, and these results suggest that the incorporation of Pmarl into the early sea urchin network has contributed to this switch.
Conserved Six3-Pax6 circuit is necessary for appropriate coelomogenesis. In sea star embryos, Delta-Notch LI segregates mesenchyme from celom. While hes $C$ expression is associated with cells fated to the celom, hesC expression is no longer detected in the mesoderm by $48 \mathrm{~h}$ (Supplementary Fig. 5e), shortly after the completion of cell type partitioning and coincident with the onset of epithelial-mesenchymal transition. The Delta-Notch LI must then "hand-off" to another set of genes to stabilize and maintain coelomic restricted gene expression. Correspondingly, this stage is also the first time that we observe the expression of pax6 and eya in this territory ${ }^{35}$. pax6, eya, six1/2, dach, and six3 are expressed in coelomic-fated mesoderm in both sea stars and sea urchins along with other genes from the highly conserved retinal determination gene 

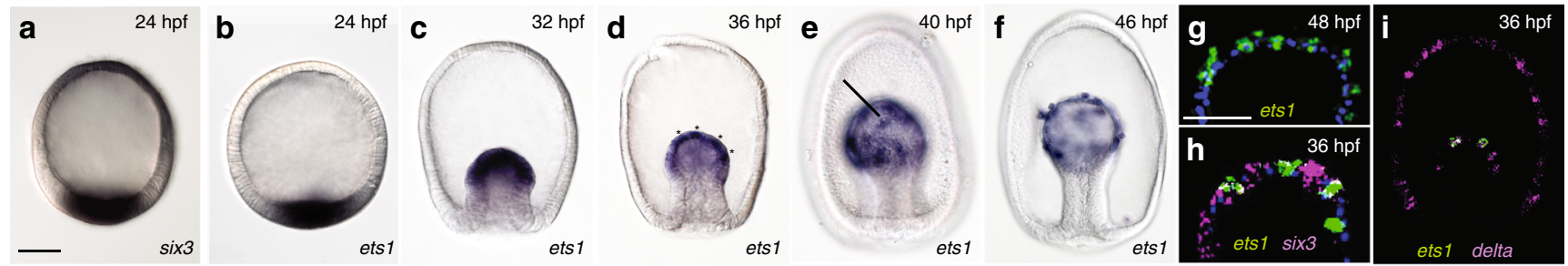

Fig. 3 Segregation of mesodermal subtypes into interleaved cells by $\mathbf{3 6} \mathbf{~ h p f . ~ S e a ~ s t a r ~ e t s ~} 1$ and six 3 transcripts are co-expressed in the vegetal mesoderm of blastula stage embryos $(\mathbf{a}, \mathbf{b})$ at $24 \mathrm{~h}$ post fertilization (hpf). The expression of the ets 1 transcript was assessed every $2 \mathrm{~h}$ from the onset of gastrulation by colorimetric WMISH. At $32 \mathrm{hpf}$ the expression of ets 1 is uniform throughout the mesoderm (c). At 36 hpf there is a discontinuity in the expression of ets 1 transcript (d, asterisks). Patches of ets1 expression become more distinct by $40-42 \mathrm{hpf}$ (e, line), and ets 1 expressing cells start to ingress beginning at 46 hpf (f). Cells expressing ets1 transcript (green) at the tip of the archenteron are adjacent to cells with no detectable ets1 expression (g), using fluorescent WMISH with a DAPI counterstain (blue), and are interleaved by cells expressing six3 transcript (magenta) (h). Cells expressing ets1 transcript also express the transcript encoding the Delta ligand (i). Data are representative of two biologically independent experiments consisting of at least ten embryos each. Scale bars represent $50 \mu \mathrm{m}$; scale bar in (a) applicable to (a-f) and (i), scale bar in ( $\mathbf{g}$ ) applicable in ( $\mathbf{g}-\mathbf{h})$, which show a magnified region at the tip of the archenteron.
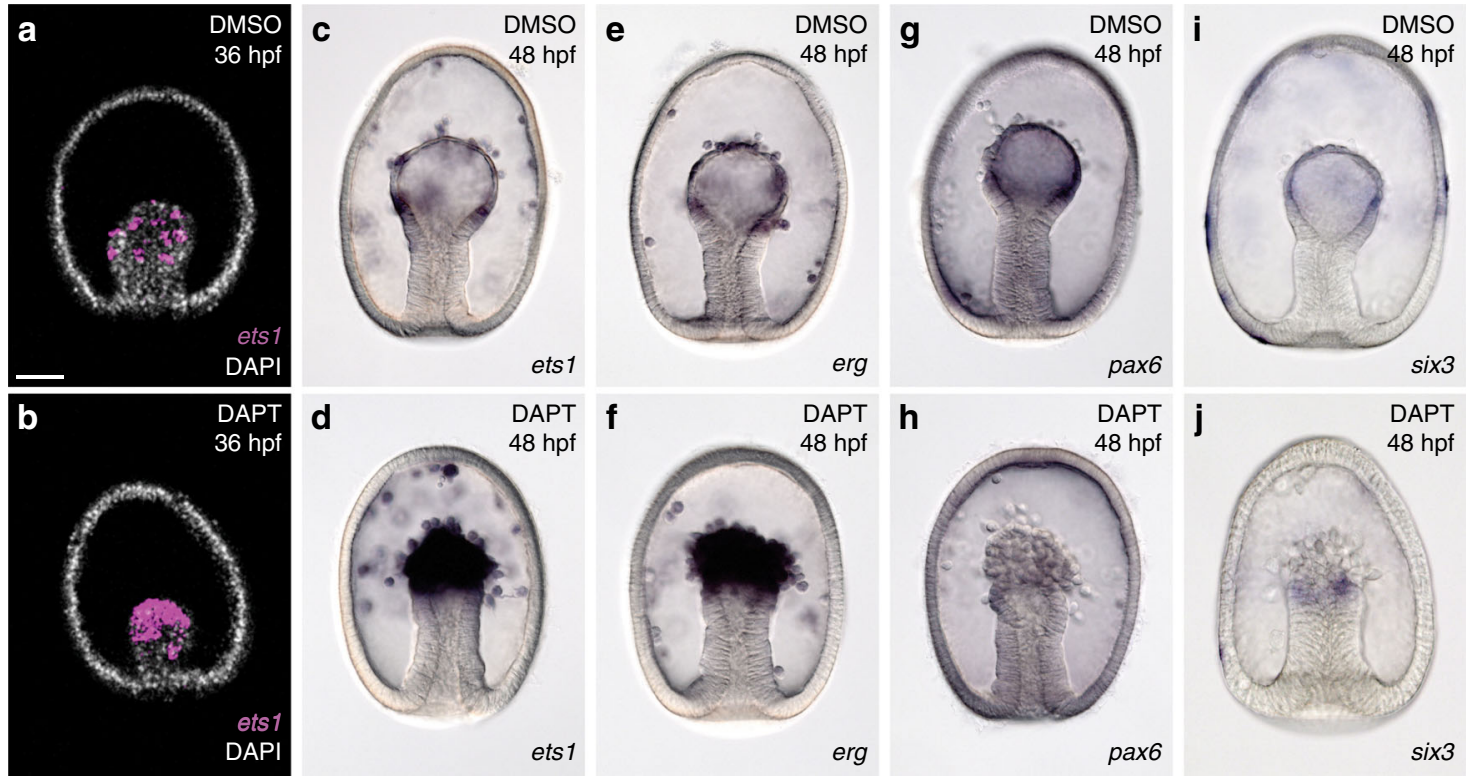

Fig. 4 Testing the lateral inhibition model of mesodermal subtype segregation. The expression of ets1 transcript (magenta) appears in a salt-and-pepper distribution throughout the mesoderm at $36 \mathrm{~h}$ post fertilization (hpf) by fluorescent WMISH (a) with DAPI stained nuclei (white). Treatment with the Notch inhibitor DAPT beginning at the 2-cell stage results in a uniform expression of ets 1 in this territory (b). By 48 hpf, the mesenchyme cells expressing ets 1 and erg $(\mathbf{c}, \mathbf{e})$ ingress into the blastocoel while cells that do not ingress express pax 6 and $\operatorname{six} 3(\mathbf{g}, \mathbf{i})$. DAPT treatment results in an increase in cells expressing ets 1 and erg $(\mathbf{d}, \mathbf{f})$ and a reduction in cells expressing pax 6 and six $3(\mathbf{h}, \mathbf{j})$. There is also a consistent morphological shift with an increase in the number of ingressing cells and a reduction in the epithelium when Notch signaling is blocked. Data shown in (c-j) are colorimetric WMISH. Data are representative of two biologically independent experiments consisting of at least ten embryos each. Scale bar represents $50 \mu \mathrm{m}$; applicable to all images in the panel.

network (RDGN) $)^{35,36}$. In the sea urchin celom, these genes are wired together in a network topology considered to be homologous to that of the $\mathrm{RDGN}^{37}$. We examined the regulatory interactions between pax6, six3, eya, dach, and sixl/2 to determine if a similar network is involved in the maintenance of sea star coelomic mesoderm. We find a similar activation of six 3 by Pax6, of six $1 / 2$ by itself, and of eya by Pax6, Six3, and Dach (Fig. 5a). Thus, these genes interact in a similar regulatory subnetwork in both sea star and sea urchin coelomic mesoderm, and this subcircuit is highly similar to the Drosophila RDGN (Fig. 5b), suggesting even deeper conservation of this network architecture. While Delta-Notch-mediated lateral inhibition is responsible for early segregation of mesenchymal and coelomic cell fates in sea stars, a Pax6 and Six3-mediated network is necessary for proper coelomogenesis after the completion of lateral inhibition (Supplementary Fig. 5).

\section{Discussion}

We present a comprehensive GRN for the specification of sea star endomesoderm; the new data presented here links the previously described GRN for the early specification of endoderm and mesoderm in the sea star to the later events during gastrulation. This GRN was produced using the same experimental approaches as those used to generate the sea urchin network ${ }^{20}$ to allow for a meaningful comparison; i.e., whole-mount in situ hybridization (WMISH) to determine spatiotemporal gene expression, and quantitative reverse transcription PCR (qRT-PCR) and WMISH in control and morpholino antisense oligonucleotide and small molecule inhibitor-induced knockdown of protein function. This network, comprising 42 nodes and 84 edges, approaches in scope the GRN for endomesoderm specification of equivalent stages in sea urchin $^{9}$, at present comprising 72 nodes and 271 edges. Although gene perturbation by morpholino knockdown has 
a
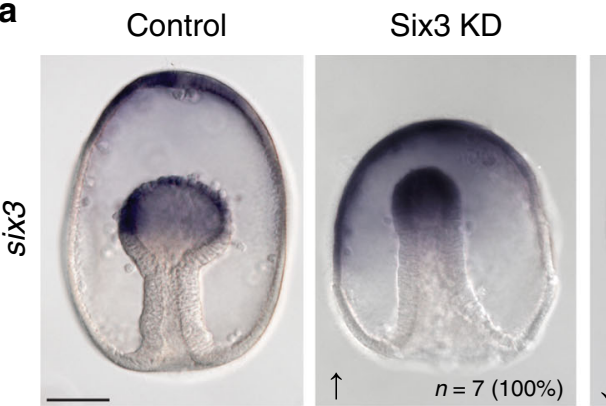

$\operatorname{Pax} 6 \mathrm{KD}$
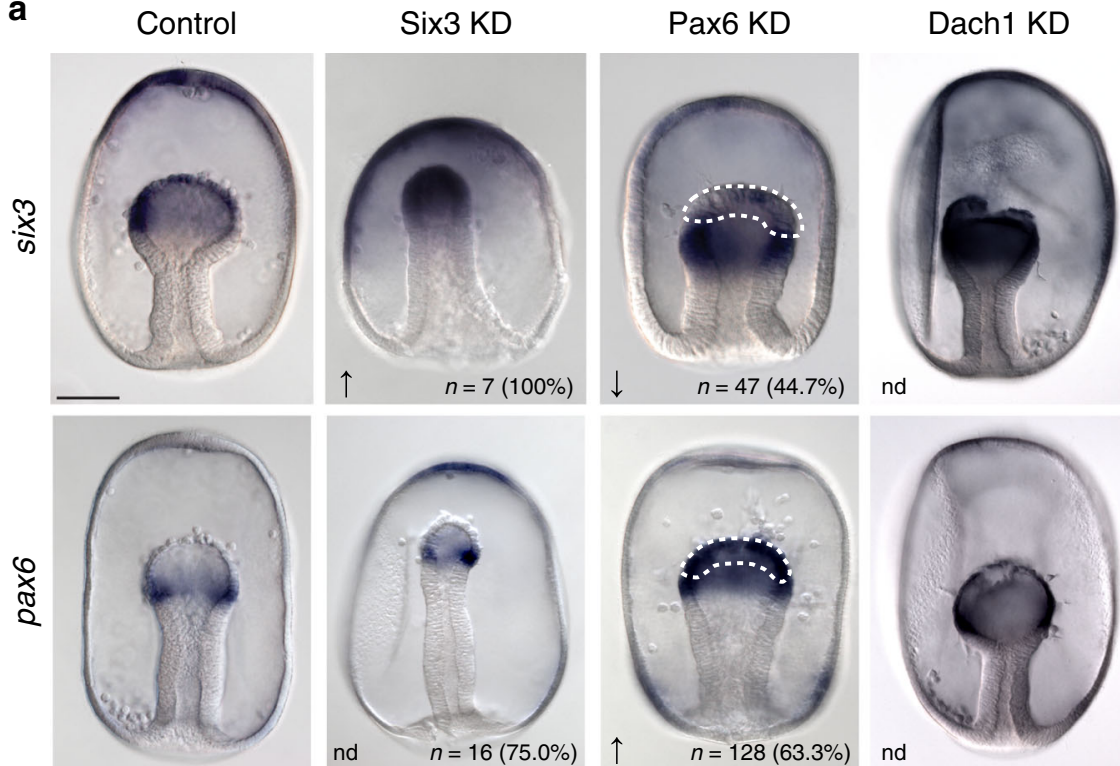

nd

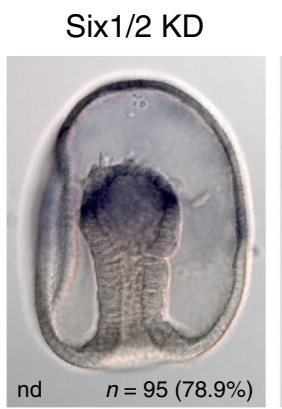

Eya KD
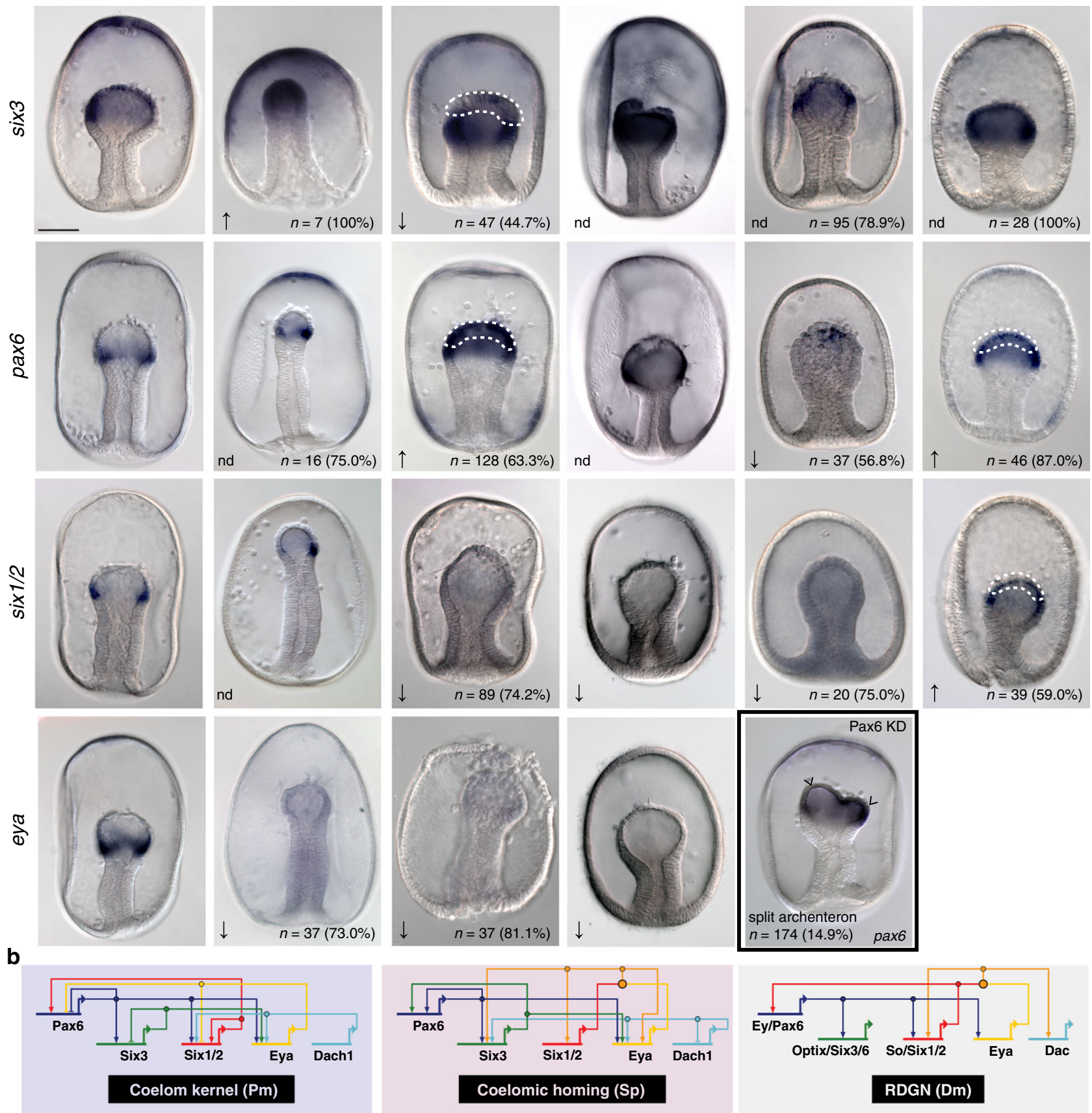

Fig. 5 Subcircuit including pax6, six3, eya, dach1, and six1/2 involved in sea star coelomogenesis. a Data shown are colorimetric WMISH using the probes indicated on the left in the conditions listed along the top. Scale bar represents $50 \mu \mathrm{m}$; applicable to all images in the panel. six 3 expression is normally distributed throughout the mesodermal bulb of the archenteron at $48 \mathrm{hpf}$, while pax6, six $1 / 2$, dach1, and eya are normally expressed at the posterior aspect of the mesodermal bulb, having been cleared earlier from anterior regions of the mesoderm. Phenotypic effect of the perturbation of each gene is indicated, including no difference (nd), increase $(\uparrow)$, decrease $(\downarrow)$. The number of embryos assessed and percent of embryos expressing the phenotype are also reported. Some reported phenotypes are localized to the top of the archenteron and are highlighted (dashed line); e.g., the effect of Pax6 knockdown on six3 expression is reported specifically for the anterior region of archenteron (dashed line). Some Pax 6 knockdown embryos exhibited a bifurcated archenteron (e.g., boxed panel, "split archenteron"). Data are representative of two biologically independent experiments consisting of at least ten embryos each and specific phenotype counts are detailed in Supplementary Table 3. These results enabled the construction of a sub-network for sea star coelomic epithelium (b) and we note a similar regulatory sub-network in both sea star and sea urchin coelomic mesoderm, which is strikingly similar to the retinal determination gene network (RDGN) in Drosophila. 
a

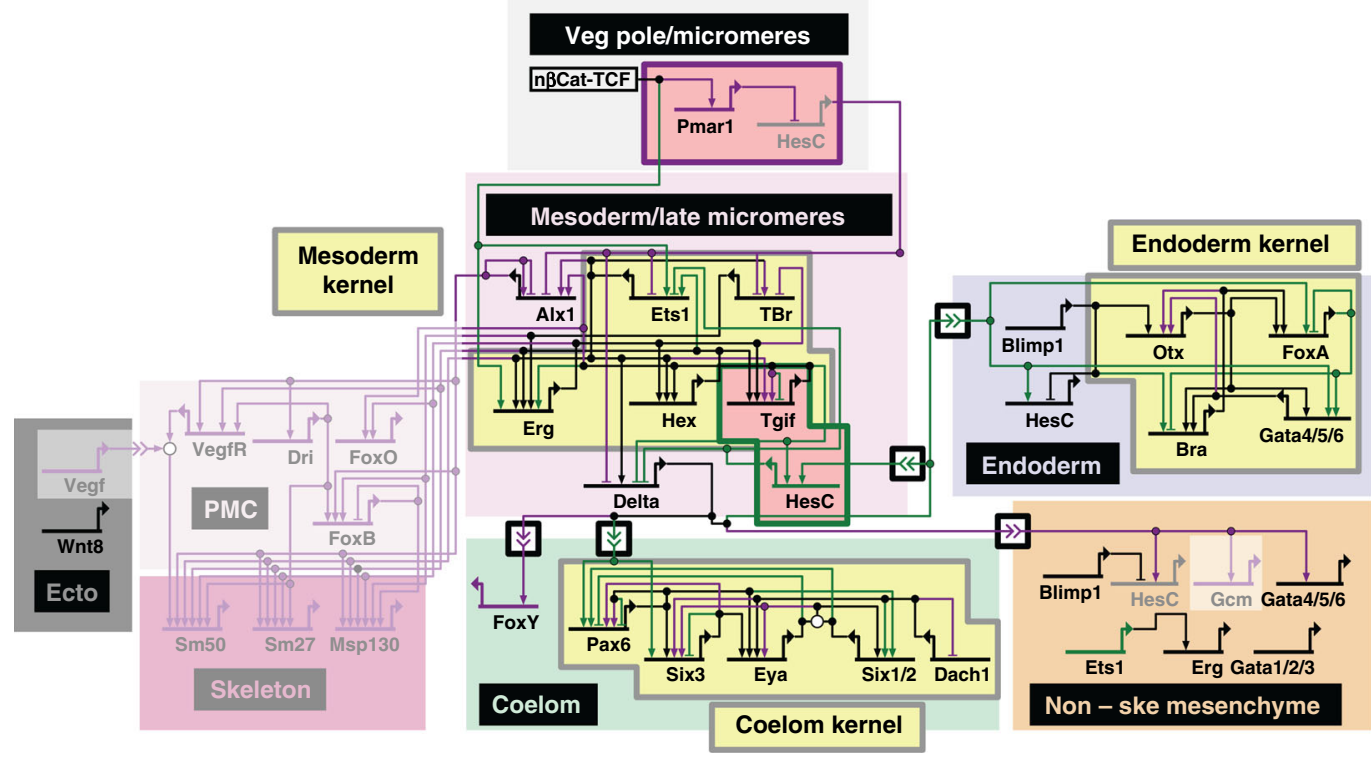

b

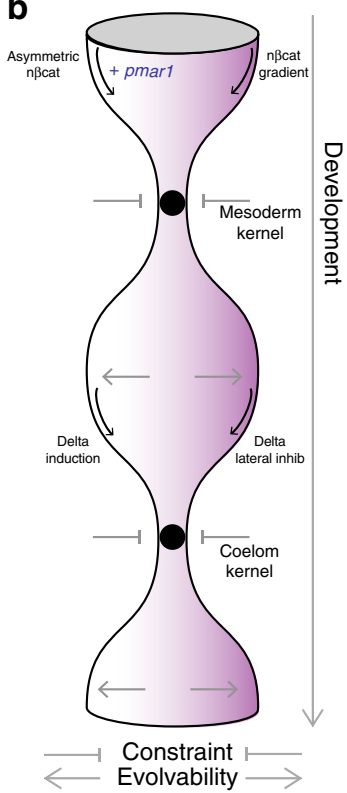

Fig. 6 Evolutionary constraint of network kernels permits alterations to the surrounding network. A synthesis of key aspects of the GRN from sea urchins and sea stars is shown (a). Genes (nodes) are shown in the territories (colored boxes) in which they are expressed. Edges show regulation by the originating upstream factor and are either positive (arrow) or repressive (bar). Signaling across cell types are indicated as double arrow heads, and Delta to Notch signals are boxed. Genes and links that are unique to sea urchin embryos are colored purple, those specific to sea stars are green, and those in common are black. Network kernels are highlighted (yellow) as are distinct sub-circuits (pink), including the sea urchin-specific double-negative gate (i.e., Pmar and $\mathrm{HesC}$, purple outline) and sea star-specific positive regulation of HesC (i.e., Tgif and HesC, green outline). Grayed out backgrounds indicate entire network circuits that are absent in sea stars. Our model of GRN evolution is depicted (b) showing that network kernels are constrained regions whereas both up and down the hierarchy the network is capable of change.

historically been a useful tool for disentangling these networks, it is important to recognize that the links drawn are largely provisional until fully borne out by independent perturbation methods (e.g. CRISPR) and cis-regulatory analyses to test whether the indicated interactions are indeed direct.

The summary view of these results permits a global comparison of these echinoderm GRN topologies (Fig. 6A), which are the synthesis of over a decade of work including the present study. Immediately apparent are the several distinct subcircuits found in common between these networks. Common modules include ets 1, erg, hex, and tgif in the mesoderm ${ }^{38,39}$; otx, gatae, bra, and foxa in the endoderm ${ }^{40,41}$; and pax6, eya, six 3 , dach, and six $1 / 2$ in the celom (Fig. $5^{37}$ ). In contrast, entire subcircuits present in sea urchins, i.e., $d r i, f o x b$, and vefgr that direct batteries of skeletogenic differentiation genes in the sea urchin micromeres, are entirely absent from the sea star network. This comparison also reveals that similarly regulated subcircuits are highly positively cross regulated, in keeping with the previous definition of network kernel ${ }^{42}$. It was previously suggested that such kernels would be found in early development, as they function downstream of maternally derived and transient signals, to stabilize gene expression needed to specify distinct embryonic territories ${ }^{43}$. A stabilizing function is thought to be derived from the intracircuit positive regulatory feedback. Here we show that these kernels appear throughout the GRN and are not limited to only early development. Preliminary experimental analyses from other species of echinoderms ${ }^{15,44-46}$ suggest these kernels are present in multiple species and thus represent a genuinely conserved, rather than convergent, feature of GRNs. The mechanistic basis for the evolutionary stability of these subcircuits remains unclear and it will be important to define additional such network motifs to begin to understand whether the observed stability is a cause or a consequence of the observed highly recursive regulatory wiring of these motifs ${ }^{42}$
From these data we propose a model of how changes in the GRN are incorporated while maintaining an overall network stability. We have detailed how the network incorporates novel circuitry into early development, in this example, the Pmar1HesC double-negative gate. These networks use the same signaling pathways at the same places in the GRN but utilize different signaling modalities of the pathways; the networks use binary versus dosage dependence of $\mathrm{n} \beta$-catenin ${ }^{47}$, and Delta induction versus Delta-Notch lateral inhibition. We argue that changes in GRNs, such as the introduction of novel genes or subcircuits, that lead to switching between alternate, stable modes of signaling pathways may be a common source of evolutionary change in these GRNs. The disruption caused by such changes would be limited if they are surrounded by stabilization features. Indeed, despite this transition in Delta-Notch signaling, we find that the GRNs in both taxa converge to a conserved regulatory subcircuit that directs the fate of coelomic mesoderm. Therefore, in contrast to previous expectations, evolutionarily stable network kernels that were proposed to function to lock down early developmental regulatory states ${ }^{2}$, are not restricted according to network hierarchy. We propose instead that these network subcircuits act as stabilizing features throughout development, functioning as developmental checkpoints through which embryogenesis must transit (Fig. 6B).

\section{Methods}

Animal and embryo handling. Adult Patiria miniata were obtained from the southern coast of California, USA (Pete Halmay or Marinus Scientific) and were used to initiate embryo cultures following the protocol by Cheatle Jarvela and Hinman ${ }^{48}$. Briefly, testes and ovaries dissected from adult $P$. miniata and oocytes and sperm were isolated. Oocytes were allowed to mature in artificial seawater (ASW) plus $10 \mu \mathrm{M}$ 1-methyladenine (Spectrum Chemical, product \# M3096) for $45 \mathrm{~min}$ prior to fertilization. Embryos were cultured in ASW at $16^{\circ} \mathrm{C}$ with occasional mixing by agitation. 
Whole-mount staining. Embryos were fixed and in situ hybridization was performed following the protocol of Hinman, Nguyen and Davidson ${ }^{49}$. Briefly, embryos were fixed in a solution of $4 \%$ paraformaldehyde in MOPS-fix buffer (0.1 M MOPS pH 7.5, $2 \mathrm{mM}$ $\mathrm{MgSO} 4,1 \mathrm{mM}$ EGTA, and $800 \mathrm{mM} \mathrm{NaCl}$ ) for 90 minutes at $25^{\circ} \mathrm{C}$ and transferred to a solution of $70 \%$ ethanol for long term storage at $-20^{\circ} \mathrm{C}$. In situ hybridization experiments were performed using digoxigenin-labeled antisense RNA probes hybridized at $60^{\circ} \mathrm{C}$. Probes were designed using gene model sequence predictions from legacy.echinobase.org ${ }^{50,51}$. Detection was performed using an anti-digoxigenin APconjugate antibody (Roche Cat\# 11093274910) followed by an NBT/BCIP reaction (Roche). For two-color FISH, a second dinitrophenyl-labeled antisense RNA probe was hybridized simultaneously ${ }^{35}$ and probes were detected using both anti-digoxigenin POD-conjugate antibody (Roche Cat\# 11207733910, RRID:AB_514500) and anti-DNP HRP-conjugate antibody (Perkin Elmer Cat\# FP1129) followed by tyramide signal amplification (Perkin Elmer). Images of colorimetric whole-mount specimens were taken using a Leica DMI 4000B microscope equipped with a Leica DFC 420C camera and fluorescent specimens were photographed using a Zeiss LSM 880 scanning laser confocal microscope. At least two independent biological replicate experiments were performed for each in situ staining experiment, examining the pattern of at least 10 specimens per replicate.

Quantitative PCR. RNA was extracted using the GenElute Mammalian Total RNA Kit (Sigma-Aldrich) and DNA was removed using the DNA-free ${ }^{\mathrm{mw}}$ DNA Removal Kit (Invitrogen). Quantitative real-time PCR was performed using the qScript OneStep SYBR Green qRT-PCR Kit (QuantaBio) and the and run on an Applied Biosystems 7300 Real-Time PCR instrument. The sequence of all qPCR primers used is reported in Supplementary Table 2. Measured Ct values for reported genes were normalized to the $\mathrm{Ct}$ of an internal control lamin $2 \beta$ receptor (GenBank ID: $\left.\mathrm{KJ} 814251.1^{52}\right)$.

Perturbation of gene expression. Zygotes were injected with morpholino antisense oligonucleotides (MASOs; GeneTools) following the protocol by Cheatle Jarvela and Hinman ${ }^{48}$. For all MASOs, the GeneTools standard control MASO was injected into sibling embryos. The observed phenotype of each MASO knockdown was confirmed by injecting a second MASO designed to the same transcript. The sequence and effective concentration used for each MASO used is reported in Supplementary Table 1 . Notch perturbations were achieved by bathing embryos in $32 \mu \mathrm{M}$ DAPT ${ }^{28}$ or dimethyl sulfoxide as a control from the two-cell stage. WMISH was performed on at least three independent sets of perturbed embryos. At least ten embryos were assessed in each replicate and phenotypes were counted and a summary is reported in Supplementary Table 3. Quantitative measures of perturbation were achieved by performing $\mathrm{qPCR}$ on perturbed compared with control siblings. Each assay was performed on at least two qPCR replicates in each of two biological replicates. Log2-transformed fold-change values between control and experimental groups are shown.

Gene regulatory network construction. The GRN model depicting sea star endomesoderm was constructed using BioTapestry 53,54 . The network was constructed by reviewing literature, spanning the years 2003-2019, which describes both embryonic gene expression and gene regulation in Patiria miniata. The experimental evidence supporting each node and edge is provided in Supplementary Data 1 and all references to work cited in the GRN experimental evidence utilized are herein cited $19,35,38,41,45,47,52,55-63$. The expression and regulatory linkages are included as reported and have been ordered according to the embryonic chronology and spatially arranged into appropriate territories. A summary of these findings is presented in Supplementary Fig. 1, and a dynamic and interactive model of a more detailed network is hosted on the web at http://grns.BioTapestry.org/ PmEndomes for further and more fine-grained exploration of the GRN ${ }^{64}$. Additionally, a BioTapestry.btp file is included in the supplementary materials (Supplementary Data 2) and can be viewed using the BioTapestry desktop application available for download at http://www.BioTapestry.org. A brief guide of how to navigate the BioTapestry user interface is provided (Supplementary Fig. 2). We expect that the online model will continue to be updated in the future to capture changes to the network.

There are three principal temporal subdivisions that span 10-24 hpf, 25-34 hpf, and $35-50 \mathrm{hpf}$, the breakpoints relating to major embryonic milestones, i.e., the distinction of mesoderm from endoderm at $\sim 24 \mathrm{hpf}$ and subsequently the split between mesenchymal and coelomic-fated mesoderm at $\sim 35 \mathrm{hpf}$. The BioTapestry user can select which of these models to view by clicking on it in the left-hand panel of the viewer. The models are organized in a hierarchy, with the top-level Full Genome model showing all nodes and links present in all the submodels. The three submodels, representing the three principal temporal subdivisions listed above, summarize the behavior of the network in the various modeled developmental domains that exist for that time period. Below each of these models in the hierarchy are dynamic models that show hourly views (using the time slider in the lower left) for that period. Note that though the time slider is hourly, expression states between the experimental data points ( $5 \mathrm{~h}$ apart) are being interpolated. The placement of data points at five-hour intervals was based on the availability of transcriptomic data spaced roughly at these intervals ${ }^{19}$ and previous studies surveying early endomesodermal specification during five-hour intervals ${ }^{47}$.
The BioTapestry model is designed to summarize the known information about the $P$. miniata developmental GRN, obtained both from literature and from our experimental results, and it is crucial to use the interactive online version to best understand the behavior of the network. The differential temporal and spatial expression patterns of the genes in the network, as determined by experiment and known with a high degree of confidence, are depicted by showing the genes as "on" or "off" (colored or gray, respectively) in the various regions of the model at each timepoint. In nodes where variable levels of expression are crucial (viz. the gradient of nuclearized beta catenin/TCF from Mesoderm to Veg1 Ectoderm that is present in the early Endomesoderm 10-24 h summary model) the nodes are depicted using intermediate levels of gray to colored. Note that by right-clicking on a gene name and selecting Experimental Data from the pop-up menu, you can view the underlying experimental expression data for the gene, as well as experimental data supporting inputs to the gene. The edges of the network are also based upon literature and experimental results. Of course, there are many different levels of confidence that can be assigned to each edge, based upon the type of experiment, and colored diamonds below the link terminus on target genes are used to indicate confidence. The highest confidence based upon detailed cis-regulatory analysis of a target gene ${ }^{61}$, is depicted with a green diamond, see e.g., Tbr activation of otx in the GRN model. However, most links in the network are backed by the results of perturbation experiments (e.g., MASO knockdown of the source gene or drug perturbation of a signaling pathway). To ensure only high confidence links are included, we use a threshold of at least twofold change observed in a minimum of two independent perturbation experiments. We also utilize multiple MASOs targeting the same transcript to ensure specificity of the observed phenotypes (see Supplementary Table 1). While there is no guarantee that these links are in fact direct, direct edges that can be explained through an indirect path can be omitted through a parsimonious approach to adding links to the network.

Links, like nodes, are also shown as "on" or "off" in the model at each point in space and time, simply based on the expression of the source gene at that same point. Notably, this depiction says nothing explicit about the actual cis-regulatory logic that is encoded in the target gene. Just because a link is shown as colored and incident on a target gene does not mean that it has been shown to be necessary at that point in space and time to cause expression of the target gene. To make that conclusion, much more targeted experiments are required to make that claim. However, the on/off state of the target gene and the inbound links can provide clues to generate hypotheses and suggest further experiments. For example, if all the links into a gray (off) target are colored (on), that suggests that there must be other unknown inputs into the target gene.

This model is not purporting to be complete but is instead a systems-level summary of the existing state of knowledge about the causal mechanisms underpinning $P$. miniata development driven by the GRN. It is certainly missing genes, and in fact since it is heavily based on orthology to genes present within the sea urchin GRN, we expect this network is biased towards including just those transcription factors. Furthermore, it has not been validated by computational simulations, and involves no detailed modeling of the transcriptional mechanisms that control gene expression. In this regard, it is like the sea urchin network, which was first developed using gene expression and perturbation data ${ }^{9}$ many years before boolean simulations were performed to ascertain the ability of the model to explain the observed behavior ${ }^{11}$.

Reporting summary. Further information on research design is available in the Nature Research Reporting Summary linked to this article.

\section{Data availability}

The authors declare that all data supporting the findings of this study are available within the article and its supplementary information files or from the corresponding author upon reasonable request. The data used to synthesize the network models presented in this paper are summarized in an online resource hosted at http://grns.BioTapestry.org/ PmEndomes/. The interactive network visualization accessible through this URL provides detail about all the experimental evidence supporting the expression timing and localization of each node in the network as well as experimental perturbations to support network edges. Each piece of data is cited to original publications for further assessment

Received: 22 July 2020; Accepted: 9 November 2020;

Published online: 04 December 2020

\section{References}

1. Levine, M. \& Davidson, E. H. Gene regulatory networks for development. Proc. Natl Acad. Sci. USA 102, 4936-4942 (2005).

2. Peter, I. S. \& Davidson, E. H. Genomic control of patterning. Int. J. Dev. Biol. 53, 707-716 (2009)

3. Slack, J. M., Holland, P. W. \& Graham, C. F. The zootype and the phylotypic stage. Nature 361, 490-492 (1993). 
4. Levin, M. et al. The mid-developmental transition and the evolution of animal body plans. Nature 531, 637-641 (2016).

5. Domazet-Lošo, T. \& Tautz, D. A phylogenetically based transcriptome age index mirrors ontogenetic divergence patterns. Nature 468, 815-818 (2010).

6. Irie, N. \& Kuratani, S. Comparative transcriptome analysis reveals vertebrate phylotypic period during organogenesis. Nat. Commun. 2, 248 (2011).

7. Kalinka, A. T. et al. Gene expression divergence recapitulates the developmental hourglass model. Nature 468, 811-814 (2010).

8. Davidson, E. H. \& Erwin, D. H. Evolutionary innovation and stability in animal gene networks. J. Exp. Zool. B Mol. Dev. Evol. 314, 182-186 (2010).

9. Davidson, E. H. et al. A genomic regulatory network for development. Science 295, 1669-1678 (2002)

10. Peter, I. S. \& Davidson, E. H. A gene regulatory network controlling the embryonic specification of endoderm. Nature 474, 635-639 (2011).

11. Peter, I. S., Faure, E. \& Davidson, E. H. Predictive computation of genomic logic processing functions in embryonic development. Proc. Natl Acad. Sci. USA 109, 16434-16442 (2012).

12. Oliveri, P., Carrick, D. M. \& Davidson, E. H. A regulatory gene network that directs micromere specification in the sea urchin embryo. Dev. Biol. 246, 209-228 (2002).

13. Revilla-i-Domingo, R., Oliveri, P. \& Davidson, E. H. A missing link in the sea urchin embryo gene regulatory network: hesC and the double-negative specification of micromeres. Proc. Natl Acad. Sci. USA 104, 12383-12388 (2007).

14. Yamazaki, A. et al. pmarl/phb homeobox genes and the evolution of the double-negative gate for endomesoderm specification in echinoderms. Development 147, dev182139 (2020).

15. Dylus, D. V. et al. Large-scale gene expression study in the ophiuroid Amphiura filiformis provides insights into evolution of gene regulatory networks. Evodevo 7, 2 (2016).

16. Hall, M. R. et al. The crown-of-thorns starfish genome as a guide for biocontrol of this coral reef pest. Nature 544, 231-234 (2017).

17. Erkenbrack, E. M. \& Davidson, E. H. Evolutionary rewiring of gene regulatory network linkages at divergence of the echinoid subclasses. Proc. Natl Acad. Sci. USA 112, E4075-E4084 (2015).

18. Simakov, O. et al. Hemichordate genomes and deuterostome origins. Nature 527, 459-465 (2015).

19. Gildor, T., Cary, G. A., Lalzar, M., Hinman, V. F. \& Ben-Tabou de-Leon, S. Developmental transcriptomes of the sea star, Patiria miniata, illuminate how gene expression changes with evolutionary distance. Sci. Rep. 9, 16201 (2019).

20. Materna, S. C. \& Oliveri, P. A protocol for unraveling gene regulatory networks. Nat. Protoc. 3, 1876-1887 (2008).

21. Yamazaki, A., Kidachi, Y., Yamaguchi, M. \& Minokawa, T. Larval mesenchyme cell specification in the primitive echinoid occurs independently of the double-negative gate. Development 141, 2669-2679 (2014).

22. Howard-Ashby, M. et al. Identification and characterization of homeobox transcription factor genes in Strongylocentrotus purpuratus, and their expression in embryonic development. Dev. Biol. 300, 74-89 (2006).

23. Sharma, T. \& Ettensohn, C. A. Activation of the skeletogenic gene regulatory network in the early sea urchin embryo. Development 137, 1149-1157 (2010).

24. Smith, J. \& Davidson, E. H. Gene regulatory network subcircuit controlling a dynamic spatial pattern of signaling in the sea urchin embryo. Proc. Natl Acad. Sci. USA 105, 20089-20094 (2008).

25. Sherwood, D. R. \& McClay, D. R. Identification and localization of a sea urchin Notch homologue: insights into vegetal plate regionalization and Notch receptor regulation. Development 124, 3363-3374 (1997).

26. Sherwood, D. R. \& McClay, D. R. LvNotch signaling mediates secondary mesenchyme specification in the sea urchin embryo. Development 126, 1703-1713 (1999).

27. Croce, J. C. \& McClay, D. R. Dynamics of Delta/Notch signaling on endomesoderm segregation in the sea urchin embryo. Development 137, 83-91 (2010).

28. Materna, S. C. \& Davidson, E. H. A comprehensive analysis of Delta signaling in pre-gastrular sea urchin embryos. Dev. Biol. 364, 77-87 (2012).

29. Sweet, H. C., Gehring, M. \& Ettensohn, C. A. LvDelta is a mesoderm-inducing signal in the sea urchin embryo and can endow blastomeres with organizerlike properties. Development 129, 1945-1955 (2002).

30. Sjöqvist, M. \& Andersson, E. R. Do as I say, Not(ch) as I do: Lateral control of cell fate. Dev. Biol. 447, 58-70 (2019).

31. Artavanis-Tsakonas, S., Rand, M. D. \& Lake, R. J. Notch signaling: cell fate control and signal integration in development. Science 284, 770-776 (1999).

32. Powell, B. C., Passmore, E. A., Nesci, A. \& Dunn, S. M. The Notch signalling pathway in hair growth. Mech. Dev. 78, 189-192 (1998).

33. Afelik, S. \& Jensen, J. Notch signaling in the pancreas: patterning and cell fate specification. Wiley Interdiscip. Rev. Dev. Biol. 2, 531-544 (2013).

34. Bray, S. J. Notch signalling: a simple pathway becomes complex. Nat. Rev. Mol. Cell Biol. 7, 678-689 (2006).
35. Yankura, K. A., Martik, M. L., Jennings, C. K. \& Hinman, V. F. Uncoupling of complex regulatory patterning during evolution of larval development in echinoderms. BMC Biol. 8, 143 (2010).

36. Byrne, M. et al. Expression of genes and proteins of the pax-six-eya-dach network in the metamorphic sea urchin: Insights into development of the enigmatic echinoderm body plan and sensory structures. Dev. Dyn. 247, 239-249 (2018)

37. Martik, M. L. \& McClay, D. R. Deployment of a retinal determination gene network drives directed cell migration in the sea urchin embryo. Elife 4, e08827 (2015)

38. McCauley, B. S., Weideman, E. P. \& Hinman, V. F. A conserved gene regulatory network subcircuit drives different developmental fates in the vegetal pole of highly divergent echinoderm embryos. Dev. Biol. 340, 200-208 (2010).

39. Oliveri, P., Tu, Q. \& Davidson, E. H. Global regulatory logic for specification of an embryonic cell lineage. Proc. Natl Acad. Sci. USA 105, 5955-5962 (2008).

40. Davidson, E. H. et al. A provisional regulatory gene network for specification of endomesoderm in the sea urchin embryo. Dev. Biol. 246, 162-190 (2002).

41. Hinman, V. F. \& Davidson, E. H. Evolutionary plasticity of developmental gene regulatory network architecture. Proc. Natl Acad. Sci. USA 104, 19404-19409 (2007).

42. Davidson, E. H. \& Erwin, D. H. Gene regulatory networks and the evolution of animal body plans. Science 311, 796-800 (2006).

43. Erwin, D. H. \& Davidson, E. H. The evolution of hierarchical gene regulatory networks. Nat. Rev. Genet. 10, 141-148 (2009).

44. Erkenbrack, E. M. et al. Ancestral state reconstruction by comparative analysis of a GRN kernel operating in echinoderms. Dev. Genes Evol. 226, 37-45 (2016).

45. McCauley, B. S., Wright, E. P., Exner, C., Kitazawa, C. \& Hinman, V. F. Development of an embryonic skeletogenic mesenchyme lineage in a sea cucumber reveals the trajectory of change for the evolution of novel structures in echinoderms. Evodevo 3, 17 (2012).

46. Cary, G. A. \& Hinman, V. F. Echinoderm development and evolution in the post-genomic era. Dev. Biol. 427, 203-211 (2017).

47. McCauley, B. S., Akyar, E., Saad, H. R. \& Hinman, V. F. Dose-dependent nuclear $\beta$-catenin response segregates endomesoderm along the sea star primary axis. Development 142, 207-217 (2015)

48. Cheatle Jarvela, A. M. \& Hinman, V. A. method for microinjection of Patiria miniata zygotes. J. Vis. Exp. e51913 (2014). https://doi.org/10.3791/51913

49. Hinman, V. F., Nguyen, A. T. \& Davidson, E. H. Expression and function of a starfish Otx ortholog, AmOtx: a conserved role for Otx proteins in endoderm development that predates divergence of the eleutherozoa. Mech. Dev. 120, $1165-1176$ (2003).

50. Kudtarkar, P. \& Cameron, R. A. Echinobase: an expanding resource for echinoderm genomic information. (Database, Oxford, 2017).

51. Cameron, R. A., Samanta, M., Yuan, A., He, D. \& Davidson, E. SpBase: the sea urchin genome database and web site. Nucleic Acids Res. 37, D750-D754 (2009).

52. McCauley, B. S., Akyar, E., Filliger, L. \& Hinman, V. F. Expression of wnt and frizzled genes during early sea star development. Gene Expr. Patterns 13, 437-444 (2013).

53. Longabaugh, W. J. R., Davidson, E. H. \& Bolouri, H. Computational representation of developmental genetic regulatory networks. Dev. Biol. 283, 1-16 (2005).

54. Longabaugh, W. J. R., Davidson, E. H. \& Bolouri, H. Visualization, documentation, analysis, and communication of large-scale gene regulatory networks. Biochim. Biophys. Acta 1789, 363-374 (2009).

55. Annunziata, R., Martinez, P. \& Arnone, M. I. Intact cluster and chordate-like expression of ParaHox genes in a sea star. BMC Biol. 11, 68 (2013).

56. Fresques, T. M. \& Wessel, G. M. Nodal induces sequential restriction of germ cell factors during primordial germ cell specification. Development 145, dev155663 (2018)

57. Fresques, T., Zazueta-Novoa, V., Reich, A. \& Wessel, G. M. Selective accumulation of germ-line associated gene products in early development of the sea star and distinct differences from germ-line development in the sea urchin. Dev. Dyn. 243, 568-587 (2014).

58. Hinman, V. F. \& Davidson, E. H. Expression of a gene encoding a Gata transcription factor during embryogenesis of the starfish Asterina miniata. Gene Expr. Patterns 3, 419-422 (2003).

59. Hinman, V. F. \& Davidson, E. H. Expression of AmKrox, a starfish ortholog of a sea urchin transcription factor essential for endomesodermal specification. Gene Expr. Patterns 3, 423-426 (2003).

60. Hinman, V. F., Nguyen, A. T., Cameron, R. A. \& Davidson, E. H. Developmental gene regulatory network architecture across 500 million years of echinoderm evolution. Proc. Natl Acad. Sci. USA 100, 13356-13361 (2003)

61. Hinman, V. F., Nguyen, A. \& Davidson, E. H. Caught in the evolutionary act: precise cis-regulatory basis of difference in the organization of gene networks of sea stars and sea urchins. Dev. Biol. 312, 584-595 (2007).

62. Otim, O., Hinman, V. F. \& Davidson, E. H. Expression of AmHNF6, a sea star orthologue of a transcription factor with multiple distinct roles in sea urchin development. Gene Expr. Patterns 5, 381-386 (2005). 
63. Yankura, K. A., Koechlein, C. S., Cryan, A. F., Cheatle, A. \& Hinman, V. F. Gene regulatory network for neurogenesis in a sea star embryo connects broad neural specification and localized patterning. Proc. Natl Acad. Sci. USA 110, 8591-8596 (2013).

64. Paquette, S. M., Leinonen, K. \& Longabaugh, W. J. R. BioTapestry now provides a web application and improved drawing and layout tools. [version 1; peer review: 3 approved]. F1000Res. 5, 39 (2016).

\section{Acknowledgements}

We thank Dr. William Hatleberg for helpful feedback during the preparation of the manuscript. This work was supported by the Binational Science Foundation grant number 2015031 to V.F.H., the National Science Foundation grants IOS 1557431 and MCB 1715721 to V.F.H. and the National Institute of Health grant P41HD071837 to V.F.H.

\section{Author contributions}

G.A.C., B.S.M., and V.F.H. conceived of and designed experiments. G.A.C., B.S.M., O.Z. and J.P. carried out experiments. G.A.C. and B.S.M. analyzed data and V.F.H. was instrumental in the interpretation of the results. G.A.C. and W.L. constructed the network model. G.A.C. wrote the manuscript with significant input from B.S.M. and V.F.H. All authors read and approved of the final manuscript.

\section{Competing interests}

The authors declare no competing interests.

\section{Additional information}

Supplementary information is available for this paper at https://doi.org/10.1038/s41467020-20023-4.

Correspondence and requests for materials should be addressed to V.F.H.

Peer review information Nature Communications thanks Maria Ina Arnone and the other, anonymous, reviewer(s) for their contribution to the peer review of this work. Peer reviewer reports are available.

Reprints and permission information is available at http://www.nature.com/reprints

Publisher's note Springer Nature remains neutral with regard to jurisdictional claims in published maps and institutional affiliations.

\begin{abstract}
(c) (i)
Open Access This article is licensed under a Creative Commons Attribution 4.0 International License, which permits use, sharing, adaptation, distribution and reproduction in any medium or format, as long as you give appropriate credit to the original author(s) and the source, provide a link to the Creative Commons license, and indicate if changes were made. The images or other third party material in this article are included in the article's Creative Commons license, unless indicated otherwise in a credit line to the material. If material is not included in the article's Creative Commons license and your intended use is not permitted by statutory regulation or exceeds the permitted use, you will need to obtain permission directly from the copyright holder. To view a copy of this license, visit http://creativecommons.org/licenses/by/4.0/.
\end{abstract}

(C) The Author(s) 2020 